\title{
Um Mapeamento Sistemático sobre Fatores que Podem Influenciar na Eficiência da Gamificação
}

\author{
Roberto Goulart S. Filho ${ }^{1}$, Henrique de Araújo Silva ${ }^{1}$, Ana Carolina Gondim Inocêncio ${ }^{1}$ \\ Universidade Federal de Goiás - Regional Jataí \\ BR 364, km 195, n 3800 - CEP 75801-615 - Jataí - Go - Brazil \\ \{robertogsfl, henriarasilva\}@gmail.com, anacarolinaufg@ufg.br
}

\begin{abstract}
This paper aims to help in the collection, selection and organization of information about the difficulties that can be encountered in the application of gamification strategy. The intention of this mapping is to list the main difficulties, which are reported by researchers who used this strategy, as well as possible ways of overcoming. Altogether 4,617 articles were found. When applying the inclusion and exclusion criteria, 27 papers were selected for a thorough reading which led to rejection of 12 and acceptance of 15 . With this mapping, it was possible to verify that a better planning is required in the application of the gamification strategy, with the intention to offer better interaction among the participants.
\end{abstract}

Resumo. Este artigo tem por objetivo auxiliar na coleta, seleção e organização das informações sobre as dificuldades que podem ser encontradas na aplicação da estratégia de gamificação. A intenção deste mapeamento é elencar as principais dificuldades, que são relatadas por pesquisadores que utilizaram desta estratégia, bem como as possiveis formas de superação. No total, foram encontrados 4.617 trabalhos. Ao aplicar os critérios de inclusão e exclusão foram selecionados 27 trabalhos para uma leitura minuciosa o que levou a rejeição de 12 e 15 foram aceitos. Com esse mapeamento foi possível verificar que se faz necessário um melhor planejamento na aplicação da estratégia de gamificação, com o intuito que esta possa oferecer melhor interação entre os participantes.

\section{Introdução}

Por muito tempo os profissionais da área de educação tiveram que conviver com a apatia e falta de interesse dos alunos na sala de aula, era uma realidade da qual a maioria massiva não conseguia escapar [Engel 2000]. Em meados de 2010, uma estratégia de interação denominada Gamificação surge com o propósito de aumentar o engajamento, dos participantes da estratégia, utilizando elementos de jogos eletrônicos (mecânicas, estratégias, pensamentos) fora do contexto dos games [Fardo 2013]. A Gamificação não envolve necessariamente atividades com jogos eletrônicos, mas a aplicação da lógica dos jogos em diferentes contextos, como o contexto escolar [Fiqueiredo et al. 2015].

A estratégia de Gamificação se constitui na utilização da mecânica dos jogos em ambiente não-jogo, criando espaços de aprendizagem mediados pelo desafio, pelo prazer e entretenimento. A principal finalidade de trazer elementos do ambiente jogo para o ambiente não-jogo é motivar os indivíduos à ação, auxiliar na solução de problemas, promover a aprendizagem de uma forma que não seja maçante para o aluno e promover 
VII Congresso Brasileiro de Informática na Educação (CBIE 2018)

Anais do XXIX Simpósio Brasileiro de Informática na Educação (SBIE 2018)

o engajamento e a vontade de aprender de uma forma que é familiar e prazerosa para a maioria dos jovens do mundo moderno.

Porém, deve-se tomar cuidado com a utilização da Gamificação. Sabe-se que a Gamificação funciona, mas não sempre. Em Hamari et al. [Hamari et al. 2014], os autores realizaram uma pesquisa analisando os trabalhos na área da Gamificação à fim de descobrir se ela funciona. A conclusão dos autores foi que ela de fato funciona mas existem alguns problemas a serem levados em consideração, como a falta de interesse com o passar do tempo, também é apresentado que muitos participantes, não necessariamente são jogadores e não se interessam pela metodologia. Mesmo os trabalhos que julgaram a experiência de gamificação, aplicada e observada, como funcional, isso não quer dizer que ela atingiu de forma positiva 100\% dos alunos [Hamari et al. 2014].

Esse problema também foi identificado por Berkling e Thomas [Berkling and Thomas 2013]. Os autores realizaram a aplicação de uma forma de Gamificação no curso de Engenharia de Software na Universidade de Baden, Alemanha. Eles julgaram o processo como um fracasso, quando analisaram os resultados. Os autores concluíram que existem alguns enganos sendo cometidos por quem opta por utilizar a Gamificação, pois perceberam que não conseguiram alcançar os objetivos desejados perante seus alunos por não saberem o que realmente os motiva e qual as particularidades de cada um.

É por essa razão que vários autores destacam a importância de conhecer o perfil dos alunos para se aplicar uma das várias formas de Gamificação mais adequada para o público em questão. O autor Kumar [Kumar 2013], estabelece que um passo crucial para o sucesso da estratégia de Gamificação é entender o jogador e o seu contexto de uso. Sendo assim, este mapeamento sistemático tem a intenção de identificar os principais fatores que influenciam na aplicação da estratégia de interação denominada gamificação, com o objetivo de auxiliar aos interessados em aplicar esta estratégia a alcançarem melhores resultados com o seu uso.

Serão explorados fatores que podem levar ao fracasso da estratégia de Gamificação na educação superior. Sobretudo, o enfoque são os aspectos da Gamificação em cursos de Computação. A Gamificação se encaixa como uma estratégia de interação que auxilia nos processos de ensino e de aprendizagem da Informática na Educação, juntamente com tecnologias vestíveis, tablets, learning analytics e MOOCs (cursos massivos gratuitos e on-line) [Klock et al. 2015], [Borges et al. 2015], [Johnson et al. 2015].

Este mapeamento sistemático está estruturado da seguinte forma: a seção 2 apresenta o conceito do mapeamento sistemático, tal como as etapas que devem ser realizadas para concretizá-lo: a definição das questões de pesquisa, a busca e seleção dos trabalhos, a extração dos dados e resultados obtidos. A seção 3 analisa esses resultados com o intuito de responder as questões de pesquisa definidas. Por último, a seção 4 descreve a conclusão deste trabalho.

\section{Metodologia}

O foco deste mapeamento sistemático é, principalmente, a identificação dos fatores que podem levar ao fracasso da estratégia de interação gamificação, a fim de responder questões de pesquisa que auxiliem na obtenção de melhores resultados aos interessados em utilizar esta estratégia. O autor Keele [Keele 2007] afirma que é necessária uma 
VII Congresso Brasileiro de Informática na Educação (CBIE 2018)

Anais do XXIX Simpósio Brasileiro de Informática na Educação (SBIE 2018)

metodologia bem definida para identificar, analisar e interpretar os estudos relevantes, existentes na literatura, a fim de responder determinadas questões de pesquisa.

Este mapeamento sistemático foi dividido em etapas de acordo com as concepções de Petersen et al. [Petersen et al. 2008], são elas: (i) Definição das questões de pesquisa, (ii) Execução da pesquisa por estudos primários relevantes, (iii) Extração e síntese de dados e (iv) Análise dos dados, conforme demonstrado nas próximas seções.

\subsection{Definição das questões de pesquisa}

A questão central que motivou esse mapeamento foi: $\mathrm{O}$ que leva a estratégia de interação denominada Gamificação a ser ineficiente ou mesmo um fracasso em alguns casos? Para responder a essa questão, cinco questões de pesquisa mais específicas foram definidas:

- Questão 1: Que tipos de dificuldades são encontradas na utilização da estratégia de interação denominada gamificação?

- Questão 2: O perfil dos participantes da gamificação influencia no êxito da aplicação desta estratégia de interação?

- Questão 3: Existe, na literatura, perfis já estabelecidos para classificar os vários tipos de participantes da estratégia de gamificação de acordo com suas preferências e afinidades quando se trata de jogos?

- Questão 4: Quais estratégias podem ser utilizadas para definir em qual perfil o participante da estratégia de interação denominada gamificação se encaixa?

\subsection{Pesquisa de Estudos Primários}

Após terem sido definidas as questões de pesquisa, definiu-se as fontes de busca. Sendo a Gamificação um assunto com grande enfoque no mundo todo, optou-se por uma pesquisa mais abrangente. Por essa razão, a pesquisa foi realizada de forma manual nas seguintes fontes de busca: Google Acadêmico (https://scholar.google.com.br/); Periódicos capes (http://www.periodicos.capes.gov.br/); IEEE (https://www.ieee.org/index.html).

Os idiomas definidos para busca e seleção dos artigos foram português e inglês, no período de 2006 à 2017. As palavras chave que compõem a busca são (em português e inglês, respectivamente): Gamificação, Gamification, dificuldade, difficulties, problemas, problems, falha, failure, sucesso, success, efetividade, effectiveness, aplicação, application, educação, education, aprendizagem, learning, aluno, student, perfil e profile.

A string de busca foi gerada a partir da combinação das palavras chave e foram divididas pelos idiomas da seguinte forma:

- Português: Gamificação AND (dificuldade OR problemas OR falha OR sucesso OR efetividade OR aplicação OR educação OR aprendizagem OR perfil) AND aluno.

- Inglês: Gamification AND (difficulties OR problems OR failure OR success OR effectiveness OR application OR education OR learning OR profile) AND student.

Os seguintes critérios de inclusão foram definidos e aplicados na seleção dos trabalhos relevantes: $O$ trabalho deve estar escrito em português ou inglês; a versão completa do trabalho deve estar disponível na web; o trabalho deve versar sobre a estratégia de interação denominada gamificação aplicada à educação com enfoque nos principais 
VII Congresso Brasileiro de Informática na Educação (CBIE 2018)

Anais do XXIX Simpósio Brasileiro de Informática na Educação (SBIE 2018)

obstáculos enfrentados na aplicação dessa estratégia; ou o trabalho deve versar sobre o perfil de estudantes/jogadores e maneiras de classificar os alunos nesses perfis; e o trabalho deve ter ano de publicação posterior ou igual a 2006. Os Critérios de Exclusão consistiram na negação ou complemento dos critérios de inclusão, além de verificar trabalhos que são réplicas, priorizando o trabalho mais recente.

Inicialmente, utilizando apenas as strings de busca, foram encontrados 4.617 artigos. Foi realizada uma triagem, a qual se deu pela leitura do título dos trabalhos em conjunto com a aplicação dos critérios de inclusão e exclusão para cada um dos trabalhos recuperados. Após essa primeira triagem, restaram 200 trabalhos. Uma segunda triagem foi realizada, a qual o resumo dos 200 trabalhos restantes foram lidos e os critérios de inclusão e exclusão reaplicados. Após a segunda triagem, restaram 27 trabalhos.

Com a leitura completa e minuciosa desses 27 trabalhos, 12 foram rejeitados, por não se adequarem ao critério de exclusão que versa sobre os perfis dos participantes e dificuldades de aplicação da estratégia de gamificação, e 15 foram aceitos. Por fim, realizou-se a extração dos dados, nesta etapa as informações necessárias para responder às questões de pesquisa foram extraídas, analisadas e interpretadas posteriormente.

\section{Resultados e Discussões}

Nessa seção, serão abordadas as duas últimas fases deste mapeamento sistemático: (iii) Extração e síntese dos dados e (iv) Análise dos dados. As questões de pesquisa serão relacionadas aos trabalhos selecionados na triagem, juntamente com uma análise e discussão dos resultados.

\subsection{Questão 1: Que tipos de dificuldades são encontradas na utilização da estratégia de interação denominada gamificação?}

No trabalho de Berkling e Thomas [Berkling and Thomas 2013], os autores aplicaram a gamificação na disciplina de Engenharia de Software de um curso de Ciência da Computação na Universidade de Baden, Alemanha. A disciplina foi toda transformada em um jogo com ambiente virtual onde os alunos teriam metas a completar até o fim da disciplina. Os autores concluíram que a experiência de gamificação foi um fracasso.

Eles chegaram a algumas conclusões que mostram algumas fragilidades da gamificação, são elas:

- Estudantes não são necessariamente jogadores: Talvez seja comum pensar que um estudante de Ciência da Computação (ou qualquer estudante) seja necessariamente um jogador, o que não é verdade. No trabalho em questão os pesquisadores descobriram que apenas $18 \%$ dos estudantes jogavam diariamente.

- Poucos estudantes jogam pelo que eles chamam de "Hard fun": "Hard fun" é o nome que foi dado aos jogadores que gostam de jogos que envolvem autonomia, estratégia, oportunidades de desafio e resolução de problemas. Geralmente a gamificação, na educação, traz esse tipo de jogo onde o aluno tem que resolver problemas e colocar o raciocínio em prática. A conclusão alcançada pelos autores é que são poucos os que gostam desse tipo de jogo, a maioria prefere jogos mais simples, jogos para passar o tempo ou relaxar. 
VII Congresso Brasileiro de Informática na Educação (CBIE 2018)

Anais do XXIX Simpósio Brasileiro de Informática na Educação (SBIE 2018)

- Jogos não são eficientes - estudantes são: Os autores descobriram que a maioria dos estudantes tiveram que estudar paras as provas da mesma forma que estudariam se a estratégia de gamificação não tivesse sido aplicada. Com isso concluíram que a maioria dos elementos da gamificação foram desnecessários para os estudantes.

- Estudantes não estão prontos para essa ideia: Com a pesquisa, os autores obtiveram que $23 \%$ dos alunos acharam a ideia útil se passar por algumas pequenas mudanças, $20 \%$ declararam que a gamificação só funcionará se passar por mudanças significantes e os últimos $25 \%$ acharam que a ideia é inútil.

- Auto regulamento/planejamento não é uma habilidade de todos: Nesse tópico os autores concluíram que nem todos os alunos tem a disciplina para participar da experiência de gamificação de forma completa. Muitos não têm a organização necessária ou a organização para tal.

Os autores Hakulinen et al. [Hakulinen et al. 2013] conduziram um experimento que agregou elementos da gamificação em um ambiente virtual de aprendizagem denominado TRAKLA2. Elementos como medalhas foram colocados no ambiente com a intenção de mudar o comportamento dos estudantes frente ao ensino. Os autores recolheram os $\log s$ do sistema, após o uso dos alunos, e fizeram uma análise do comportamento dos mesmos.

Foi concluído que os elementos da gamificaçao tiveram um impacto positivo na maioria dos estudantes, porém alguns problemas também foram identificados. Um deles foi o incentivo de um comportamento indesejado entre os alunos, que tiveram atitude muito competitiva e descuidada em frente aos desafios do sistema. Além disso, o uso dos elementos da gamificação não foi suficiente para estimular o aprendizado completo, sendo assim os alunos tiveram que estudar para as avaliações da mesma forma.

No trabalho de Figueiredo et al. [Fiqueiredo et al. 2015], os autores fizeram a análise de vários trabalhos publicados na área da gamificação e identificaram em seus estudos algumas questões recorrentes. Uma delas é o fato de educadores não terem o hábito de interagir com jogos eletrônicos, em sua maioria. É claro que isso não é uma regra, mas o Jogador está longe de ser uma classe dominante entre os atuais educadores, principalmente no Brasil. Um outro ponto analisado por eles é a falta de uma base sólida do que é uma prática pedagógica gamificada, ou seja, a falta de conhecimento do que é gamificação, seus benefícios e como utiliza-la.

Outro autor que identificou uma situação em que o professor teve problemas em aplicar uma estratégia de Gamificação foi Fardo [Fardo 2013]. Em seu trabalho, o autor analisou a Gamificação em um contexto geral e, por fim, em um contexto específico. Ele concluiu que, em geral, a Gamificação é benéfica para o aprendizado e tende a crescer e se popularizar entre os educadores. No contexto especifico, o autor observou uma experiência de Gamificação que teve como maior fragilidade a falta de experiência do professor em aplicar e administrar a experiência. Para Fardo [Fardo 2013], a utilização da Gamificação no contexto da sala de aula requer uma boa dose de compreensão por parte dos professores, e que esse é particularmente um dos grandes desafios em nossa realidade.

Em Brühlmann [Brühlmann 2017], o autor diz que ainda não é claro sob que circunstâncias a gamificação vai ser efetiva. Isso se deve ao que o autor chama de baixo rigor e baixa generalização dos estudos na área da gamificação. 
VII Congresso Brasileiro de Informática na Educação (CBIE 2018)

Anais do XXIX Simpósio Brasileiro de Informática na Educação (SBIE 2018)

No trabalho de Hamari et al. [Hamari et al. 2014], os autores fizeram uma análise de vários trabalhos na área da gamificação com a intenção de descobrir se ela realmente funciona. Eles concluíram que ela funciona mas apresenta algumas fragilidades e a maior delas é que a estratégia chamada de gamificação tem um efeito positivo nos usuários por um curto período de tempo. Esse período acontece no início da prática, depois os alunos vão perdendo o interesse e deixando de participar gradualmente.

\subsection{Questão 2: O perfil dos participantes da gamificação influencia no êxito da aplicação desta estratégia de interação?}

No trabalho de Kim [Kim 2011], a autora explica a gamificação de um ponto de vista do designer social. Para ela, pontos, medalhas e placares não são o suficiente para criar uma experiência de jogo em um ambiente não-jogo pois, eles são meramente elementos de retorno. Ela insiste que entender o estilo social e o nível de perícia do usuário é de crucial importância. Ela conclui que os elementos da gamificação devem ser usados de acordo com o perfil de usuário especifico.

Para Nicholson [Nicholson 2015], a gamificação não será eficiente se não for planejada baseado em um bom entendimento dos usuários e do contexto de uso. Para o autor, uma análise do contexto de uso também é um pré-requisito e por fim o perfil do usuário como um todo.

O autor Kumar [Kumar 2013], estabelece que um passo crucial para o sucesso da gamificação é entender o jogador e o seu contexto de uso. Para ele, o sucesso da sua gamificação depende do claro entendimento do perfil do usuário. Conhecer o seu público é de vital importância para qualquer projeto e a gamificação não é exceção.

\subsection{Questão 3: Existem, na literatura, perfis já estabelecidos para classificar os vários tipos de participantes da estratégia de gamificação de acordo com suas preferências e afinidades quando se trata de jogos?}

Em Kumar [Kumar 2013], o autor cita o trabalho de Bartle [Bartle 1996] para classificar os jogadores em quatro perfis: Matadores, Exploradores, Socializadores e Relizadores. Bartle [Bartle 1996] criou esses perfis baseado em pessoas que gostam de jogar, jogam por hobby.

Tunanen e Hamari [Tuunanen and Hamari 2012] fazem uma análise dos trabalhos que surgiram depois de Bartle [Bartle 1996] para classificar os tipos de jogadores. Cada autor faz uma alteração nos arquétipos, adicionando ou alterando alguns da forma que acharam mais pertinente de acordo com sua pesquisa. Mas, no fim todos estão classificando jogadores e alunos não são necessariamente jogadores [Berkling and Thomas 2013].

No trabalho de Heeter et al. [Heeter et al. 2011], os autores criaram novos perfis de jogadores com enfoque em estudantes e realizaram uma pesquisa em uma universidade à fim de classificar os alunos nos perfis criados. A pesquisa consistiu de um questionário, respondido pelos alunos, com perguntas que os encaixariam em certo perfil dependendo de suas respostas. Os perfis de Heeter et al. [Heeter et al. 2011], apesar de serem pensados para estudantes, não foram pensados para ambientes gamificados.

Marczewski [Marczewski 2015] criou uma tipologia de perfis baseada em Bartle e descreve os jogadores conforme suas motivações. Ao contrário das demais classificações 
VII Congresso Brasileiro de Informática na Educação (CBIE 2018)

Anais do XXIX Simpósio Brasileiro de Informática na Educação (SBIE 2018)

descritas (que são voltadas para jogos), essa tipologia descreve seis tipos específicos para ambientes gamificados.

No trabalho realizado por Marczewski [Marczewski 2015], são descritos seis perfis de jogadores, são eles: Achievers, Disruptors, Free Spirits, Philanthropists, Players e Socialisers. Essa tipologia foi baseada no trabalho de Bartle [Bartle 1996] e descreve os jogadores conforme suas motivações. Ao contrário das demais tipologias que são voltadas para Jogos, essa tipologia descreve perfis específicos para ambientes gamificados. A seguir, serão descritos cada um dos perfis definidos por Marczewski.

O perfil Achiever contempla os jogadores motivados pelo domínio. Esses jogadores tentam aprender novas coisas e melhorar a si mesmos através da superação de desafios. Disruptors são jogadores motivados pela mudança. Para isso, eles tentam desestruturar o sistema e forçar uma mudança para causar desordem, seja de forma direta ou através de outros jogadores [Marczewski 2015].

Free Spirits são jogadores motivados pela autonomia e pela auto-expressão. Eles tendem a explorar o ambiente como lhe convém ou de construir e descobrir coisas novas. Philanthropists são jogadores motivados pelo propósito. São jogadores altruístas, que tendem a deixar de lado seus ideais pelos outros e gostam de ajudar os demais jogadores sem esperar recompensa por isso [Marczewski 2015].

Players são jogadores motivados pelas recompensas. Essa pessoa espera recompensas em troca de qualquer ato realizado por eles. O ideal, para este tipo de jogador, é que ambiente forneça recompensas ao mesmo tempo em que tenta motivá-los a desenvolver habilidades, explorar o sistema, ajudar e se relacionar com os demais jogadores. Socialisers são jogadores motivados pelos relacionamentos. A troca de relações entre jogadores é o principal fator de motivação para essa pessoa. Para isso, eles interagem com outros usuários e visam a criação de conexões sociais [Marczewski 2015].

\subsection{Questão 4: Quais estratégias podem ser utilizadas para definir em qual perfil o participante da estratégia de interação denominada gamificação se encaixa?}

No artigo de Pastor et al. [Pastor et al. 2007], os autores propõem a utilização da técnica de análise de dados chamada: Análise latente de perfil (Latent profile analysis - LPA). Os autores defendem que essa é a técnica mais confiável para análise de dados e tentam mostrar que outras técnicas como a análise de cluster (cluster analysis) e regressão múltipla (multiple regression) são menos confiáveis se comparadas a LPA. Os autores utilizam essa técnica para classificar os diferentes tipos de metas de alunos em determinado perfis.

O trabalho de Breasfelean et al. [Bresfelean et al. 2008], propõem a utilização de técnicas de análise de cluster (cluster analysis) e agrupamento de dados (data clustering) para descobrir o perfil doas alunos que reprovam em disciplinas na faculdade.

O trabalho de Carmona et al. [Carmona et al. 2007], os autores propõem a utilização de aprendizagem de máquina (machine learning) para aprender sobre os usuários e classifica-los. A ideia é deixar o aluno interagir com o sistema, nesse meio tempo o sistema iria aprendendo sobre o aluno até conseguir identificar seu perfil dentro de alguns já pré-estabelecidos.

Uma forma mais simples de classificar uma pessoa em seu perfil é o questionário proposto por Marczewski [Marczewski 2015]. Seu questionário é especifico para clas- 
VII Congresso Brasileiro de Informática na Educação (CBIE 2018)

Anais do XXIX Simpósio Brasileiro de Informática na Educação (SBIE 2018)

sificar a pessoa em um de seus perfis, mas pode ser adaptado e utilizado com outras tipologias. O questionário de Marczewski possui 24 perguntas, sendo 4 perguntas para cada perfil. Essas perguntas devem ser respondidas conforme uma escala de Likert de 5 pontos (onde 1 equivale a "Discordo Totalmente" e 5 equivale a "Concordo Totalmente"). A escala de Likert é um instrumento para mensurar a realidade sobre um objeto em estudo. Como resultado, o questionário retorna o percentual de cada tipo de jogador do aluno, definindo seu tipo predominante e os tipos secundários.

\section{Considerações finais}

O mapeamento sistemático, apresentado nesse artigo, teve como foco as fragilidades e dificuldades enfrentadas pela estratégia de interação denominada gamificação. As questões de pesquisa foram criadas com o intuito de descobrir o porquê dessas dificuldades e as possíveis formas de contorna-las.

A questão 1 mostra várias dificuldades enfrentadas pela gamificação são apresentadas e explanadas, concluindo que existem problemas ao se utilizar dessa estratégia de forma arbitrária. Analisando as questões de pesquisa 2 e 3 , é possível concluir que descobrir o perfil do público alvo pode ser a estratégia mais eficiente para que uma experiência de gamificação seja o mais eficiente possível, quando se trata de alcançar máximo engajamento dos alunos e, consequentemente, máxima aquisição de conhecimento pelos mesmos. Existem alguns perfis já definidos na literatura, mas a maioria trata de perfil de jogadores com exceção da tipologia de Marczewski. A classificação dos alunos em perfis pode ser feita utilizando técnicas avançadas de análise de dados, utilizando questionários ou por meio de aprendizado de máquina, como mostra a questão de número 5.

Esta pesquisa tem por objetivo auxílio de pesquisadores e educadores que desejam utilizar a gamificação, seja para estudo ou aplicação. Um ponto importante, que foi possível observar com este mapeamento, é que um melhor planejamento e análise dos participantes da estratégia de gamificação poderá culminar em um maior grau de interação e compreensão do conteúdo a ser trabalhado.

\section{Referências}

Bartle, R. (1996). Hearts, clubs, diamonds, spades: Players who suit muds. Journal of MUD research, 1(1):19.

Berkling, K. and Thomas, C. (2013). Gamification of a software engineering course and a detailed analysis of the factors that lead to it's failure. In Interactive Collaborative Learning (ICL), 2013 International Conference on, pages 525-530. IEEE.

Borges, V. A., Nogueira, B. M., Barbosa, E. F., et al. (2015). Uma análise exploratória de tópicos de pesquisa emergentes em informática na educação. Revista Brasileira de Informática na Educação, 23(2):85-96.

Bresfelean, V. P., Bresfelean, M., Ghisoiu, N., and Comes, C.-A. (2008). Determining students' academic failure profile founded on data mining methods. In Information Technology Interfaces, 2008. ITI 2008. 30th International Conference on, pages 317322. IEEE.

Brühlmann, F. (2017). The Effects of Framing in Gamification: A Study of Failure. Springer. 
VII Congresso Brasileiro de Informática na Educação (CBIE 2018)

Anais do XXIX Simpósio Brasileiro de Informática na Educação (SBIE 2018)

Carmona, C., Castillo, G., and Millán, E. (2007). Discovering student preferences in elearning. In Proceedings of the international workshop on applying data mining in e-learning, pages 33-42.

Engel, G. I. (2000). Pesquisa-ação. Educar em Revista, (16):181-191.

Fardo, M. L. (2013). A gamificação aplicada em ambientes de aprendizagem. RENOTE, 11(1).

Fiqueiredo, M., Paz, T., and Junqueira, E. (2015). Gamificação e educação: um estado da arte das pesquisas realizadas no brasil. In Anais dos Workshops do Congresso Brasileiro de Informática na Educação, volume 4, page 1154.

Hakulinen, L., Auvinen, T., and Korhonen, A. (2013). Empirical study on the effect of achievement badges in trakla 2 online learning environment. In Learning and Teaching in Computing and Engineering (LaTiCE), 2013, pages 47-54. IEEE.

Hamari, J., Koivisto, J., and Sarsa, H. (2014). Does gamification work?-a literature review of empirical studies on gamification. In System Sciences (HICSS), 2014 47th Hawaii International Conference on, pages 3025-3034. IEEE.

Heeter, C., Lee, Y.-H., Medler, B., and Magerko, B. (2011). Beyond player types: Gaming achievement goal. In Proceedings of the 2011 ACM SIGGRAPH Symposium on Video Games, pages 43-48. ACM.

Johnson, L., Adams Becker, S., Estrada, V., and Freeman, A. (2015). The NMC Horizon Report: 2015 Higher Education Edition. ERIC.

Keele, S. (2007). Guidelines for performing systematic literature reviews in software engineering. In Technical report, Ver. 2.3 EBSE Technical Report. EBSE. sn.

Kim, A. (2011). Smart gamification: Seven core concepts for creating compelling experiences. Recuperado a partir de http://www. youtube. com/watch, 4.

Klock, A. C. T., da Cunha, L. F., and Gasparini, I. (2015). Um modelo conceitual para a gamificação de ambientes virtuais de aprendizagem. RENOTE, 13(1).

Kumar, J. (2013). Gamification at work: Designing engaging business software. In International Conference of Design, User Experience, and Usability, pages 528-537. Springer.

Marczewski, A. C. (2015). Even Ninja Monkeys Like to Play: Gamification, Game Thinking and Motivational Design. CreateSpace Independent Publishing Platform.

Nicholson, S. (2015). A recipe for meaningful gamification. In Gamification in education and business, pages 1-20. Springer.

Pastor, D. A., Barron, K. E., Miller, B., and Davis, S. L. (2007). A latent profile analysis of college students' achievement goal orientation. Contemporary Educational Psychology, 32(1):8-47.

Petersen, K., Feldt, R., Mujtaba, S., and Mattsson, M. (2008). Systematic mapping studies in software engineering. In EASE, volume 8, pages 68-77.

Tuunanen, J. and Hamari, J. (2012). Meta-synthesis of player typologies. In Proceedings of Nordic Digra 2012 Conference: Games in Culture and Society, Tampere, Finland. 